\title{
Carta ao editor sobre o artigo: Fixação intramedular flexível para fraturas diafisárias dos ossos do antebraço em crianças
}

\section{Letter to the Editor about the article: Intramedullary Flexible Nailing for Diaphyseal Fractures of Forearm Bones in Children}

\author{
Nicolas Gallardo-Molina1(1) \\ ${ }^{1}$ Departamento de Pesquisa, Medical School, Espiritu Santo \\ University, Samborondon, Ecuador \\ Rev Bras Ortop 2020;55(6):808.
}

\section{Caro Editor,}

0 artigo de Acharya et al $^{1}$ mostra evidências importantes sobre o tratamento cirúrgico das fraturas do antebraço em crianças, o que é preciso de acordo com estudos recentes aqui apresentados. A fixação intramedular flexível mostrou excelentes resultados, principalmente na consolidação sem danificar a placa de crescimento, retorno às atividades normais e poucas complicações. Por exemplo, Kapoor et $\mathrm{al}^{2}{ }^{2}$ em seu estudo com 50 crianças, demonstraram consolidação em todas as fraturas em aproximadamente 7 semanas e, em termos de complicações, apenas 2 pacientes necessitaram de fasciotomia para síndrome do compartimento pós-operatório e 9 pacientes relataram restrição na pronação em 20 graus.

No entanto, a revisão sistemática de Patel et $\mathrm{al}^{3}$ mostra uma comparação de complicações e resultados funcionais entre hastes intramedulares flexíveis e placas de fixação. Os resultados apresentam que não há diferenças significativas em tempo de consolidação, complicações, grau de angulação, encurtamento ou rotação. A única diferença é que a haste intramedular flexível revela melhores resultados estéticos e menor tempo de cirurgia. Portanto, a decisão final de qual método usar vai depender da experiência do cirurgião.

Por outro lado, o artigo de Peterlein et $\mathrm{al}^{4}$ fornece informações importantes para serem aplicadas em todos os pacientes submetidos a esse tipo de cirurgia, pois se concentra em avaliar os resultados a longo prazo da haste intramedular flexível nas fraturas diafisárias de antebraço em um total de 122 crianças. Os pacientes foram avaliados com a escala de deficiências no braço, ombro e mão (DASH,
Endereço para correspondência Nicolas Gallardo-Molina, MD, Research Department at Medical School of Espiritu Santo University (UEES), Via Samborondon km 1.5, Guayaquil, Guayas 092302, Ecuador (e-mail: gallardonicolasm@gmail.com).

na sigla em inglês) e com a escala de Mayo Wrist. A pontuação do DASH é composta pelas deficiências mencionadas acima, e a avaliação da realização de esportes e o trabalho. Um total de $77 \%$ atingiu uma escala DASH igual a 0 , isto é, esse número de pacientes não relatou queixas após o procedimento. Além disso, a escala de Mayo Wrist é uma ferramenta que avalia quatro variáveis que são dor, força de preensão, amplitude de movimento e satisfação. Este estudo relata uma escala de Mayo Wrist de 100 em 82\% $(n=74)$ dos pacientes, quer dizer, que a maioria não apresentou nenhum problema.

Baseado nisso, pode-se determinar que o procedimento cirúrgico da haste intramedular flexível não apresenta diferenças significativas em relação a outros métodos, como a placa de fixação ou tratamento conservador. Portanto, a maioria dos estudos determina que esse método dispõe de certas vantagens que favorecem a recuperação e a qualidade de vida pós-operatória do paciente.

\section{Referências}

1 Acharya B, Devkota P, Thakur A, Gyawali B. Fixação intramedular flexível para fraturas diafisárias dos ossos do antebraço em crianças. Rev Bras Ortop 2019;54(05):503-508

2 Kapoor V, Theruvil B, Edwards SE, Taylor GR, Clarke NMP, Uglow MG. Flexible intramedullary nailing of displaced diaphyseal forearm fractures in children. Injury 2005;36(10):1221-1225

3 Patel A, Li L, Anand A. Systematic review: functional outcomes and complications of intramedullary nailing versus plate fixation for both-bone diaphyseal forearm fractures in children. Injury 2014; 45(08):1135-1143
DOI https://doi.org/ 10.1055/s-0040-1709735. ISSN 0102-3616.
Copyright $\odot 2020$ by Sociedade Brasileira License terms de Ortopedia e Traumatologia. Published by Thieme Revinter Publicações Ltda, Rio de Janeiro, Brazil 